\title{
De Garengeot Hernia: A Case Report and Review of Literature
}

\author{
Aashish Shah $\cdot$ Haridarshan Sira janardhan
}

Received: 11 November 2010 / Accepted: 20 November 2012 /Published online: 9 December 2012

(C) Association of Surgeons of India 2012

\begin{abstract}
Femoral Hernia constitutes a small percentage of groin herniae,but have always been associated with significantly high morbidity. This is partly due to the difficulties in diagnosing the hernia and also due to its propensity for incarceration because of its anatomy. We report a rare case of De Garengeot Hernia which is the herniation of the appendix into a femoral hernia. While this is rare in itself, acute appendicitis in a strangulated femoral hernia is even more uncommon.
\end{abstract}

Keywords Femoral hernia $\cdot$ Acute appendicitis $\cdot$ Contrast Enhanced CT · Diagnostic laparoscopy · Laparotomy · Anatomical repair

\section{Introduction}

Femoral hernias occur just below the inguinal ligament when the abdominal contents pass through the femoral canal. Femoral hernia is relatively uncommon type, accounting for only $3 \%$ of all hernias. Whereas femoral hernias can occur in both male and female population, almost all of them develop in women because of the wider bone structure of the female pelvis [1]. Herniation of the appendix into a femoral hernia - also called De Garengeot hernia - is a rare but a well-recognized entity. It is generally an incidental

\footnotetext{
A. Shah $\cdot$ H. Sira janardhan

Department of MAS and GI Surgery, Fortis Hospital,

Bannerghatta Road,

Bangalore 560076, India

A. Shah $(\bowtie)$

76-1-1, VR Layout, 1st Phase JP Nagar,

Bangalore 560078, India

e-mail: ashlap@yahoo.com
}

finding during hernia repair. Acute appendicitis in a strangulated femoral hernia is even more uncommon. Because of its rarity, we present a case report of a 46-year-old female diagnosed to have acute appendicitis with obstruction in the femoral canal with a brief review on the literature.

\section{Case Report}

A 46-year-old female was admitted in our hospital with an acute onset of periumbilical abdominal pain of 3 days duration, associated with loose stools for 2 days. The patient also had a history of mild fever for 2 days and bilious vomiting 1 day before admission.

There were no history of hematemesis, melena, or hematochezia, and the patient denied any previous history of similar episodes. The patient had a past medical history of left hemiplegia (the right middle cerebral artery territory infarct), rheumatic heart disease, and atrial fibrillation.

The patient was on the following medications:

$$
\begin{aligned}
& \text { Acitrom tablet } 1 \mathrm{mg} \\
& \text { Restyl } 0.25 \mathrm{mg} \\
& \text { Betaloc } 25 \mathrm{mg} \\
& \text { Eltroxin } 50 \mu \mathrm{g}
\end{aligned}
$$

On admission, the patient was hemodynamically stable and in sinus rhythm. She was febrile with a temperature of $99^{\circ} \mathrm{F}$ and was mildly dehydrated. Her abdominal examination revealed features of peritonism in the right iliac fossa, right lumbar region, and suprapubic area.

There was generalized tenderness all over the abdomen. There was no palpable mass in the abdomen. Though free on both sides, the hernial orifice was tender on palpation on the right side. Bowel sounds were sluggish. 

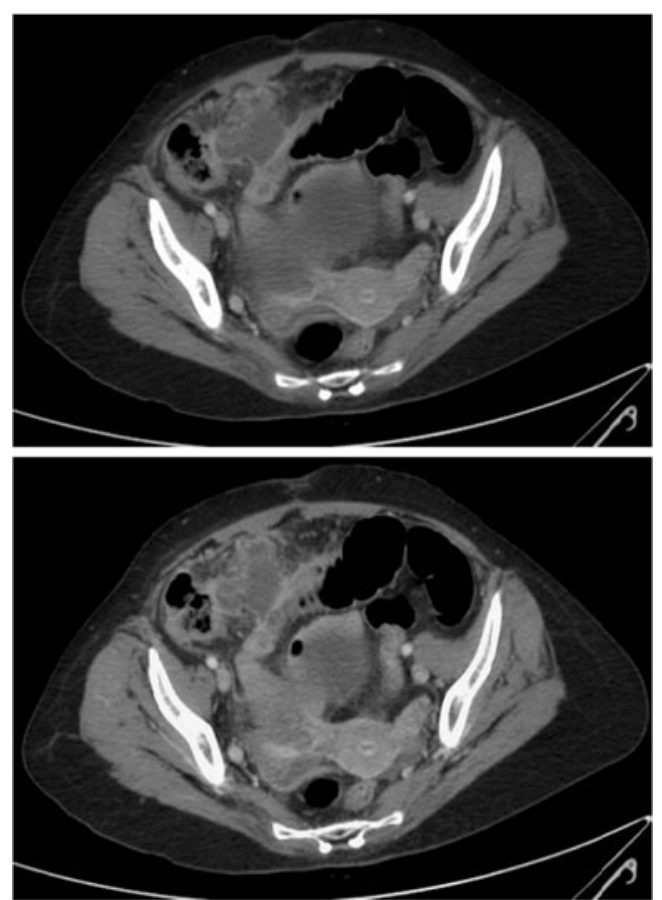

Fig. 1 CT image showing interloop abscess formation with fluid in the right iliac fossa and obstruction at the distal ileum

A clinical diagnosis of peritonitis possibly secondary to ischemic gut was made with a differential diagnosis of perforated appendicitis. Routine investigations were normal except for leukocytosis. Abdominal X-ray did not reveal any significant abnormality. A contrast computed tomographic (CT) scan of the abdomen and pelvis suggested features of small bowel obstruction at the distal ileal level, with adjacent inflammatory fluid collection in the right iliac fossa suggestive of bowel perforation with interloop abscess formation (Fig. 1).

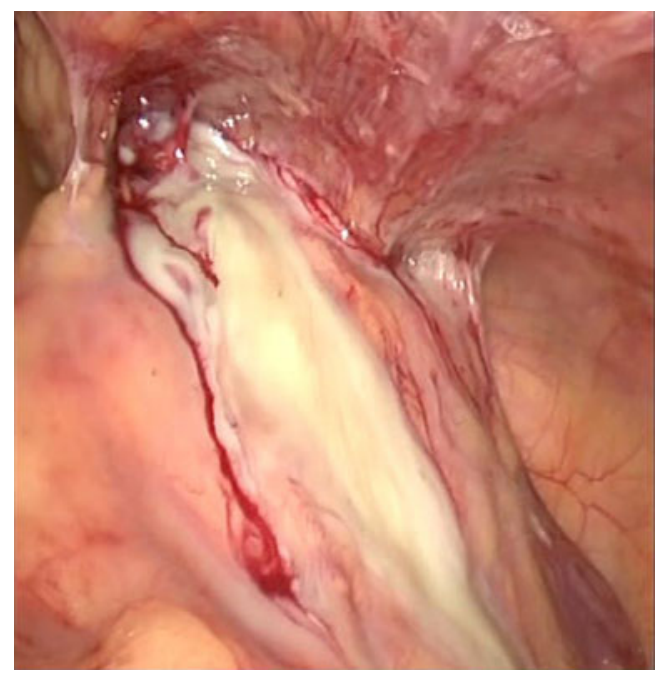

Fig. 2 Interloop adhesions with small bowel adhesions

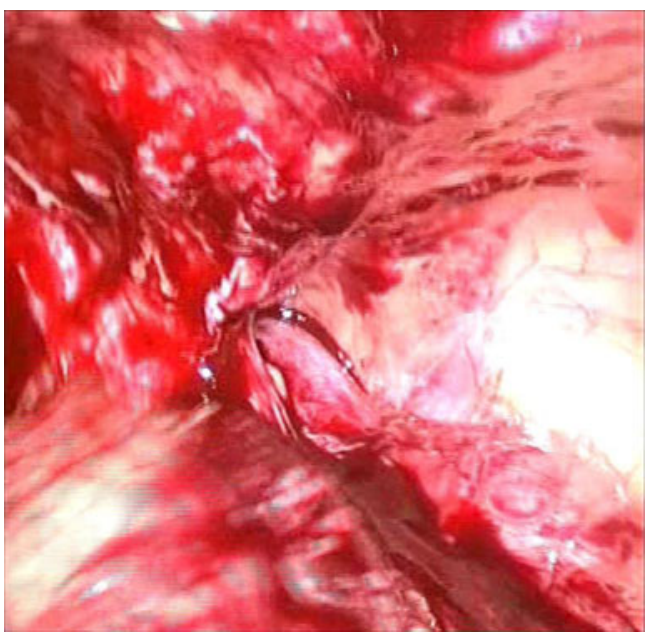

Fig. 3 Non-reducible obstructed appendix in the femoral canal

Based on the contrast-enhanced CT findings, a presumptive diagnosis of small intestinal perforation with peritonitis was made, and the patient was counseled and consented for a diagnostic laparoscopy and/or laparotomy.
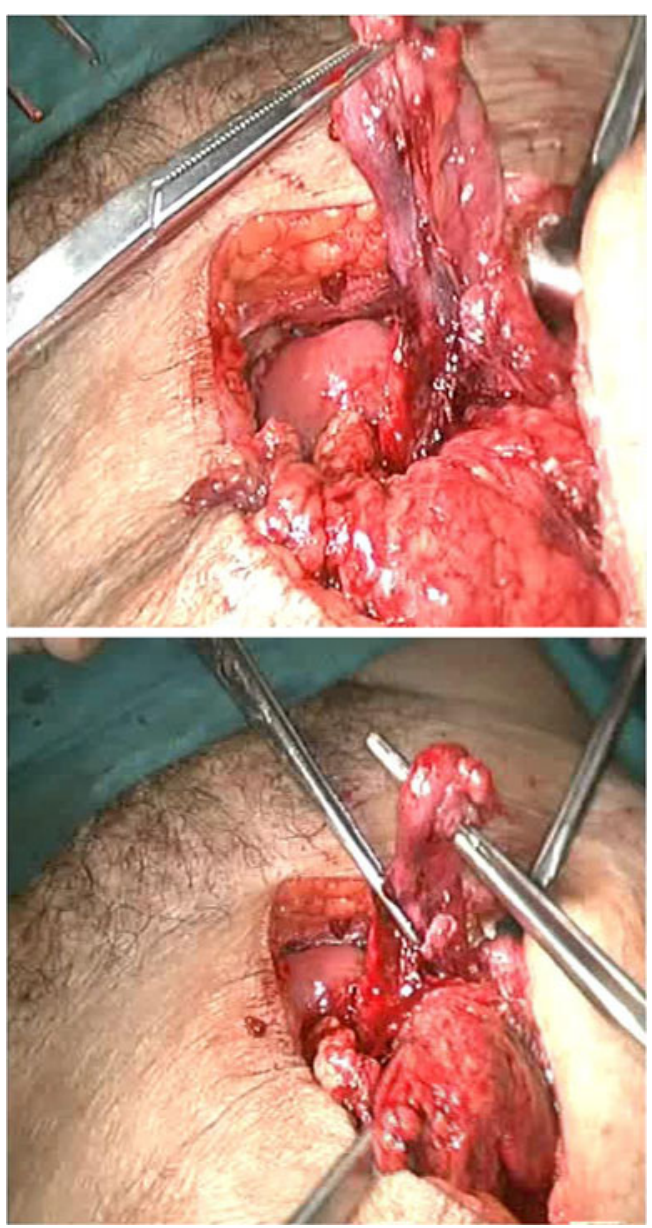

Fig. 4 Acutely kinked appendix brought out via laparotomy after releasing from the femoral canal 
Diagnostic laparoscopy revealed the following:

1. Phlegmon with small bowel loops and omentum en masse in the pelvic region extending to the right iliac fossa.

2. Interloop abscess with small bowel adhesions involving the terminal ileum and cecum (Fig. 2).

3. A non-reducible inflamed, turgid appendix with periappendiceal phlegmon was seen, entering and exiting the right femoral canal—acutely kinked in a tight "U" (Fig. 3).

Drainage of interloop abscess with adhesiolysis and thorough inspection of the small bowel loops was performed laparoscopically. Inability to reduce the herniated inflamed appendix from the femoral canal (De Garengeot hernia) necessitated conversion to a lower midline laparotomy.

Laparotomy was performed; appendix was released and reduced, and appendicectomy was performed followed by a thorough peritoneal lavage (Fig. 4). No bowel pathology could be identified.

The femoral defect was closed with three interrupted of 2.0 Prolene sutures. The midline laparotomy was closed by the mass closure technique. The patient made an uneventful postoperative recovery.

The biopsy report revealed inflammatory changes within the appendix consistent with appendicitis. No evidence of malignancy was noted.

\section{Discussion and Review of Literature}

Rene Jacques Croissant De Garengeot, a Parisian surgeon, was the first to describe the appendix in a femoral hernia sac in 1731. Femoral hernia commonly occurs in women and is considered to be a congenital defect. Because of the narrowness and rigidity of the femoral canal, the rate of incarceration in femoral hernias (14-56\%) is significantly higher than that in inguinal hernias $(6-10 \%)$, and therefore, a femoral hernia requires early surgical repair [2].

Acute appendicitis within a femoral hernia accounts for $0.13 \%$ of all cases of acute appendicitis [3]. Usually inflammation of the appendix is attributed to intraluminal obstruction or obstruction at the opening with the cecum. However, it is thought that external compression of the appendix at the neck of the hernia explains the cause of acute appendicitis in femoral hernia [4, 5]. The anatomy of the femoral canal usually prevents intraperitoneal spread of infection, and hence, the patient will not present with symptoms of peritonitis but rather local signs such as erythema and groin tenderness [5]. Femoral hernia is the groin hernia with the highest rate of incarceration and strangulation (5-20\%), therefore requiring early surgical repair. Diagnosis is usually made clinically, presenting as a groin lump.

However, fewer than two-third cases of femoral hernia are correctly diagnosed before hospital admission, and over $10 \%$ are diagnosed only at laparotomy. In our study, no bowel obstruction was noted as only the appendix was involved.

The use of imaging in these cases is controversial. CT scanning has been shown to be of benefit in some studies; however, it is quite unlikely and difficult to diagnose appendicitis within the groin mass itself [6]. The best modality of investigation and treatment is diagnostic laparoscopy and surgical exploration with resection of the appendix and repair of the femoral defect. It is not recommended to place synthetic mesh plugs within the defect because of the infective and inflammatory processes involved with appendicitis.

\section{Conclusion}

The rarity of De Garengeot hernia makes a preoperative diagnosis very difficult, if not impossible, even with a CT scan. Most often, a diagnostic laparoscopy and/or open exploration is the only way of diagnosing this condition, while at the same time allowing for a definitive procedure. Appendicectomy with thorough peritoneal toileting and an anatomical repair of the femoral canal is the procedure of choice.

\section{References}

1. Rastegari EC. Femoral hernia repair. Advameg, Inc 2009 (accessed on 12th July 2012).

2. Cordera F, Sarr MG (2003) Incarcerated appendix in a femoral hernia sac. Contemp Surg 59:35-37, accessed on 12th July 2012

3. Luchs JS, Halpern D, Katz DS (2000) Amyand's hernia: prospective CT diagnosis. J Comput Assist Tomogr 24:884-886, accessed on 12th July 2012

4. Nguyen ET, Komenaka IK (2004) Strangulated femoral hernia containing a perforated appendix. Can J Surg 47:68-69, accessed on 12th July 2012

5. D'Ambosio N, Katz D, Hines J (2006) Perforated appendix within a femoral hernia. AJR Am J Roentgenol 186:906-907, accessed on 12th July 2012

6. Zissin R, Brautbar O, Shapiro-Feinberg M (2000) CT diagnosis of acute appendicitis in a femoral hernia. Br J Radiol 73:1013-1014, accessed on 12th July 2012 